\title{
Qualidade de vida do trabalhador docente em Educação Física do estado do Paraná, Brasil
}

\author{
Quality of life of teaching in Physical Education of Parana \\ state, Brazil
}
Hudson de Resende Moreira ${ }^{1}$ Juarez Vieira do Nascimento ${ }^{2}$ Christi Noriko Sonoo Jorge Both ${ }^{4}$

1 Universidade do Vale do Itajaí. Rede Municipal de Ensino de São José, São José, SC.Brasil

2 Universidade Federal de Santa Catarina. Programa de Pós-Graduação em Educação Física. Florianópolis, SC. Brasil

3 Universidade Estadual de Maringá. Programa de Pós-Graduação em Educação Física. Maringá, PR. Brasil

4 Rede Municipal de Ensino de Florianópolis. Programa de Pós-Graduação em Educação Física. Florianópolis, SC. Brasil

Recebido em 24/01/10 Revisado em 21/04/10 Aprovado em 07/06/10
Resumo - O objetivo do estudo foi avaliar o nível de correlação entre os constructos da qualidade de vida no trabalho (QVT) e do estilo de vida (EV) dos docentes de Educação Física da rede pública estadual de ensino do Paraná. A amostra foi constituída por 654 docentes (299 professores do sexo masculino e 355 professores do sexo feminino), o que corresponde um erro amostral de 3,5\%. Na coleta dos dados foi aplicado um questionário sociodemográfico, a "Escala de Avaliação da Qualidade de Vida no Trabalho Percebida por Professores de Educação Física do Ensino Fundamental e Médio" e "Perfil do Estilo de Vida Individual". O teste de Qui-quadrado para grupo único e o teste de correlação de Spearman foram empregados na análise estatística dos dados. Os resultados evidenciaram que os docentes estão insatisfeitos com os salários, condições de trabalho e com o trabalho e espaço total de vida. A maioria apresentou comportamento positivo quanto ao EV, porém os componentes nutrição, atividade física e controle do estresse apresentam grande preocupação. Constatou-se que a avaliação interna da QVT obteve índices de correlação maiores que do EV. Ao serem cruzadas as duas matrizes analíticas, observaram-se níveis fracos de correlação entre os componentes e as avaliações globais dos constructos.

Palavras-chave: Qualidade de vida; Trabalho; Estilo de vida; Professor; Educação física.

Abstract - The aim of this study was to evaluate the correlation between the constructs of quality of work life (QWL) and lifestyle in Physical Education teachers from public schools in Parana State. The sample consisted of 654 teachers (299 men and 355 women), corresponding to a sampling error of 3.5\%. A sociodemographic questionnaire, "Perceived Work Quality of Life Scale by Physical Education Teachers from Elementary to High School" and "Profile of Individual Lifestyle" were used for data collection. Statistical analysis was performed using the chi-square test and Spearman's correlation test. The results showed the dissatisfaction of teachers with wages, working conditions and balance between work and leisure. Most subjects presented positive lifestyle behaviors, but the components nutrition, physical activity and stress management were a matter of concern. Internal evaluation of QWL yielded higher correlation coefficients than lifestyle. When comparing the two analytical matrices, low levels of correlation were observed between components and overall assessment of the constructs.

Key words: Quality of life; Work; Lifestyle; Teacher; Physical education. 


\section{INTRODUÇÃO}

A percepção de Qualidade de Vida (QV) pode variar de indivíduo para indivíduo, independente do grupo social a que pertença. Além disso, diferentes fatores que permeiam o cotidiano das pessoas são comumente associados à QV, nomeadamente a longevidade, a satisfação laboral, a relação com a família, o salário, as condições urbanas, a espiritualidade e o lazer. A percepção do indivíduo em relação à QV pode sofrer influência de fatores socioambientais e de fatores individuais, que juntos, geram uma sensação de bem ou mal estar e, consequentemente, boa ou má QV'1.

Enquanto os parâmetros socioambientais estão relacionados à educação, ao meio ambiente, à assistência médica, ao transporte, à segurança, à moradia e ao lazer, os parâmetros individuais dizem respeito à hereditariedade e ao estilo de vida $(\mathrm{EV})^{1}$. O EV aborda questões referentes aos hábitos alimentares, à atividade física, aos relacionamentos com seus pares, ao comportamento preventivo em relação a doenças e atitudes sociais e ao controle do estresse.

As investigações que reportam associações entre a qualidade de vida no trabalho (QVT) e o EV durante a carreira docente de professores de Educação Física descrevem situações distintas de percepção de satisfação com a QVT em diferentes momentos da carreira. Estas constatações podem estar relacionadas ao plano de carreira implementado nos diferentes estados ${ }^{2,3}$. Além disso, pesquisas têm evidenciado problemas com a saúde física e mental de docentes, favorecendo o afastamento temporário, ou mesmo, definitivo da profissão ${ }^{4,5}$.

A baixa remuneração, o desprestígio social, o acúmulo de funções, as condições de trabalho desfavoráveis e a elevada carga horária laboral, aliados ao aumento da violência escolar, a má relação com a direção escolar e com seus pares e a problemas relacionados ao estresse crônico no ambiente laboral (Síndrome de Burnout, mal estar docente ou Síndrome do Esgotamento Profissional) têm sido elementos identificados em investigações que retratam condições desfavoráveis à prática docente e que dão indícios de influenciar negativamente a percepção dos professores de Educação Física em relação à QVT e ao comportamento referente ao $\mathrm{EV}^{6-11}$.

Diante do fato de que os estudos são mais sugestivos do que conclusivos sobre esta temática, torna-se pontual investigar o nível de correlação entre os constructos da QVT e do EV de professores de Educação Física. Assim, o objetivo do estudo foi de avaliar o nível de correlação existente entre as dimensões da QVT (parâmetro socioambiental) com os componentes do EV (parâmetro individual) e suas correlações internas conforme a percepção e comportamento dos docentes de Educação Física da rede pública estadual de ensino do Paraná, Brasil.

\section{PROCEDIMENTOS METODOLÓGICOS}

O estudo caracteriza-se como sendo descritivo correlacional, cuja população foi composta por 4.770 professores de Educação Física, efetivos da rede estadual de ensino do Paraná. O processo de seleção da amostra ocorreu em duas fases. A primeira fase buscou estratificar proporcionalmente as mesorregiões do estado, para que, na segunda, fase houvesse um processo de seleção por conglomerados. Destaca-se que os Núcleos Regionais de Educação (NREs) foram considerados os conglomerados neste estudo.

Participaram do estudo professores efetivos de Educação Física na rede pública estadual de ensino; que não exercessem cargo de gestão ou remanejamento (readaptação) e atuantes frente aos alunos na escola. Após esta seleção, os professores foram convidados a participarem desta investigação, de maneira voluntária, garantindo-lhe sigilo de suas informações e assegurando que os dados obtidos seriam utilizados com propósito investigativo.

Para o cálculo da amostra, foi utilizado, inicialmente, um erro amostral de 5\%, com intervalo de confiança de $95 \%$. Devido ao universo populacional, evidenciou-se a necessidade de se obter uma amostra esperada de 356 professores a partir de fórmula contida no Programa Epi-info 3.5.1. Porém, com o envio de 2.310 questionários, obteve-se amostra alcançada de 654 (299 professores do sexo masculino e 355 professores do sexo feminino) questionários validos (28,31\%), estabelecendo-se novo erro amostral de $3,5 \%$.

Instrumentos para a coleta de dados

Dois instrumentos foram utilizados na coleta de dados. O primeiro instrumento foi a "Escala de Avaliação da Qualidade de Vida no Trabalho Percebida por Professores de Educação Física do Ensino Fundamental e Médio" (QVT-PEF) ${ }^{12}$, o qual obteve um alfa de Cronbach de 0,94, o que é considerado como excelente. Este instrumento está estruturado em oito componentes conforme a proposta de Walton ${ }^{13}$ (1973): a) remuneração e compensação; b) condições de trabalho; c) oportunidade imediata para uso e desenvolvimento de capacidades humanas; d) oportunidade futura de 
crescimento e segurança; e) integração social na organização do trabalho; f) constitucionalismo na organização de trabalho; g) trabalho e espaço total de vida; h) relevância social da vida no trabalho. A escala de avaliação é do tipo Likert de 1 a 7, onde: (1) discordo totalmente; (2) discordo bastante; (3) discordo um pouco; (4) não concordo nem discordo; (5) concordo um pouco; (6) concordo bastante; (7) concordo totalmente.

$O$ segundo instrumento utilizado na pesquisa foi o "Perfil do Estilo de Vida Individual"14, o qual obteve uma consistência interna considerada razoável (alfa de Cronbach de 0,78) ${ }^{15}$. O instrumento é composto por 15 perguntas abrangendo cinco componentes: nutrição, atividade física, comportamento preventivo, relacionamentos e controle do estresse. A escala de avaliação é do tipo Likert de 0 a 3, onde: (0) absolutamente não faz parte do seu estilo de vida; (1) às vezes corresponde ao seu comportamento; (2) quase sempre verdadeira no seu comportamento; (3) a afirmação é sempre verdadeira no seu dia-a-dia; faz parte do seu estilo de vida.

\section{Processo de coleta de dados}

Para a coleta de dados, foram enviados via correspondência postal, envelopes contendo os instrumentos e os termos de consentimento livre e esclarecido aos NREs do Estado do Paraná. Estes núcleos distribuíram, via mala direta, estes envelopes aos diretores das escolas que, por sua vez, redistribuíram aos professores de Educação Física. Após o preenchimento dos questionários pelos docentes, as escolas retornaram via mala direta os envelopes, contendo os instrumentos e os termos de consentimento livre e esclarecido aos NREs que, por sua vez, encaminharam-nos aos pesquisadores via correspondência postal.
Destaca-se que esta investigação obteve aprovação do projeto junto ao Comitê de Ética em Pesquisa com Seres Humanos da Universidade Federal de Santa Catarina, por meio do Parecer no 036/07.

\section{Análise dos dados}

Inicialmente, os dados foram categorizados conforme as equações de ponderação de $\operatorname{Lemos}^{16}$, que se utilizou dos mesmos instrumentos em seu estudo, delimitando percepções de insatisfeito, indeciso e satisfeito para a QVT e comportamentos positivo, intermediário e negativo para o EV.

Após a categorização, realizou-se uma ponderação da amostra para que houvesse um equilíbrio amostral de cada região na análise estatística. Os testes estatísticos empregados na análise foram o qui-quadrado para grupo único para observar as tendências de opiniões nas dimensões/componentes e avaliações globais da QVT e do EV (referência no teste 33,3\%) e, para avaliar a correlação entre as variáveis, utilizou-se correlação de Spearman. $\mathrm{Na}$ análise, utilizou-se o programa estatístico SPSS, versão 15.0, com um intervalo de confiança de 95\%.

Por fim, destaca-se que os índices de correlação foram classificados de acordo com os critérios estabelecidos por Mitra e Lankford ${ }^{17}$ : fraca correlação, valores entre 0,20 e 0,39; moderada correlação, valores entre 0,40 e 0,59; e forte correlação, valores acima de 0,60.

\section{RESULTADOS}

Em relação à distribuição das frequências das dimensões e da avaliação global da QVT (Tabela 1), observa-se que os professores investigados apresentaram uma tendência de satisfação na maioria (7 entre 8) das dimensões avaliadas $(\mathrm{p} \leq 0,001)$.

Tabela 1. Distribuição das frequências das dimensões e da avaliação global da qualidade de vida no trabalho.

\begin{tabular}{lcccc}
\hline \multirow{2}{*}{ Dimensões } & \multicolumn{3}{c}{ Qualidade de Vida no Trabalho } & p-valor* \\
\cline { 2 - 3 } & $\begin{array}{c}\text { Insatisfeitos } \\
(\%)\end{array}$ & Indecisos (\%) & Satisfeitos (\%) & \\
\hline Remuneração e compensação & 44,0 & 35,4 & 20,6 & $<0,001$ \\
Condições de trabalho & 31,6 & 29,2 & 39,2 & $<0,001$ \\
Oportunidade imediata para uso e desenvolvimento das & 4,1 & 21,4 & 74,5 & $<0,001$ \\
capacidades humanas & 5,1 & 23,4 & 71,4 & $<0,001$ \\
Oportunidade futura de crescimento e segurança & 10,7 & 38,2 & 51,1 & $<0,001$ \\
Integração social na organização do trabalho & 5,9 & 17,7 & 76,4 & $<0,001$ \\
Constitucionalismo na organização do trabalho & 21,5 & 30,6 & 47,9 & $<0,001$ \\
Trabalho e espaço total de vida & 5,3 & 17,2 & 77,6 & $<0,001$ \\
Relevância social da vida no trabalho & 8,4 & 26,3 & 65,3 & $<0,001$ \\
\hline Avaliação global da qualidade de vida no trabalho & & & & \\
\hline
\end{tabular}

* p-valor estimado através do Qui quadrado para grupo único (referência das proporções = 33,3\%) 
Observa-se que as dimensões que demonstraram maiores níveis de satisfação foram relevância social da vida no trabalho $(77,6 \%)$, constitucionalismo na organização do trabalho $(76,4 \%)$ e oportunidade imediata para uso e desenvolvimento das capacidades humanas $(74,5 \%)$. Por outro lado, os maiores índices de insatisfação foram constatados nos componentes remuneração e compensação (44,0\%), condições de trabalho $(31,6 \%)$ e trabalho e espaço total de vida (21,5\%).

Quanto ao estilo de vida (Tabela 2), constatou-se que os professores relataram comportamento positivo na maioria (4 de 5) dos componentes do EV e na avaliação global $(\mathrm{p} \leq 0,001)$. No entanto, os componentes nutrição, atividade física e controle do estresse apresentam grande preocupação devido aos elevados percentuais de perfil negativo e intermediário.

Além disso, constatou-se que os assuntos relacionados ao comportamento preventivo $(78,8 \%)$ e relacionamentos $(76,6 \%)$ são os fatores mais positivos do estilo de vida. Entretanto, os componentes nutrição $(33,9 \%)$, atividade física $(24,6 \%)$ e controle do estresse $(22,4 \%)$ são aqueles nos quais existe maior comportamento negativo entre os investigados.
Em relação às correlações entre as dimensões e avaliação global da QVT (Tabela 3), constatou-se que a avaliação global da QVT obteve forte correlação com as dimensões condições de trabalho $\left(r_{s} 0,66\right)$; oportunidade imediata para uso e desenvolvimento das capacidades humanas $\left(r_{s}\right.$ 0,65); integração social na organização do trabalho $\left(r_{s} 0,62\right)$; constitucionalismo na organização do trabalho $\left(r_{s} 0,62\right)$ e relevância social da vida no trabalho $\left(\mathrm{r}_{\mathrm{s}} \mathrm{0}, 64\right)$. Destaca-se, também, as correlações moderadas com as dimensões remuneração e compensação $\left(r_{s} 0,45\right)$, oportunidade futura de crescimento e segurança $\left(r_{s} 0,57\right)$ e com o trabalho e espaço total de vida $\left(r_{s} 0,49\right)$.

Observa-se que as dimensões relacionadas à remuneração e compensação e ao trabalho e espaço total de vida apresentaram as correlações mais fracas com as demais dimensões ( $r_{s}$ entre 0,19 e 0,38). Entretanto, a dimensão relevância social da vida no trabalho $\left(\mathrm{r}_{\mathrm{s}}\right.$ entre 0,22 e 0,52) e a dimensão constitucionalismo na organização do trabalho ( $r_{s}$ entre 0,25 e 0,53), demonstraram as correlações mais fortes com as demais dimensões que compõem o constructo da QVT.

Os resultados encontrados não revelaram a existência de fortes correlações entre as dimensões

Tabela 2. Distribuição das frequências das dimensões e da avaliação global do estilo de vida.

\begin{tabular}{lcccc}
\hline \multirow{2}{*}{ Componentes } & \multicolumn{3}{c}{ Estilo de Vida } & \multirow{2}{*}{ p-valor* } \\
\cline { 2 - 3 } & Negativo (\%) & Intermediário (\%) & Positivo (\%) & \\
\hline Nutrição & 33,9 & 32,4 & 33,7 & 0,579 \\
Atividade física & 24,6 & 22,7 & 52,7 & $<0,001$ \\
Comportamento preventivo & 7,1 & 14,0 & 78,8 & $<0,001$ \\
Relacionamentos & 6,6 & 17,8 & 76,6 & $<0,001$ \\
Controle do estresse & 22,4 & 26,2 & 51,4 & $<0,001$ \\
Avaliação global do estilo de vida & 7,1 & 31,8 & 61,0 & $<0,001$ \\
\hline
\end{tabular}

* p-valor estimado através do Qui quadrado para grupo único (referência das proporções = 33,3\%).

Tabela 3. Correlação entre as dimensões e a avaliação global da qualidade de vida no trabalho.

\begin{tabular}{|c|c|c|c|c|c|c|c|c|c|}
\hline Variáveis & QVT 1 & QVT 2 & QVT 3 & QVT 4 & QVT 5 & QVT 6 & QVT 7 & QVT 8 & QVT Global \\
\hline \multicolumn{10}{|l|}{ QVT 1} \\
\hline QVT 2 & $0,38^{*}$ & & & & & & & & \\
\hline QVT 3 & $0,32 *$ & $0,47^{*}$ & & & & & & & \\
\hline QVT 4 & $0,26^{*}$ & $0,45^{*}$ & $0,38^{*}$ & & & & & & \\
\hline QVT 5 & $0,31^{*}$ & $0,44^{*}$ & $0,43^{*}$ & $0,36^{*}$ & & & & & \\
\hline QVT 6 & $0,26^{*}$ & $0,39 *$ & $0,48^{*}$ & $0,43^{*}$ & $0,53^{*}$ & & & & \\
\hline QVT 7 & $0,19 *$ & $0,32 *$ & $0,29 *$ & $0,23 *$ & $0,28^{*}$ & $0,25^{*}$ & & & \\
\hline QVT 8 & $0,22^{*}$ & $0,41^{*}$ & $0,52^{*}$ & $0,42^{*}$ & $0,43^{*}$ & $0,47^{*}$ & $0,33^{*}$ & & \\
\hline QVT Global & $0,45^{*}$ & $0,66^{*}$ & $0,65^{*}$ & $0,57^{*}$ & $0,62^{*}$ & $0,62^{*}$ & $0,49 *$ & $0,64^{*}$ & \\
\hline
\end{tabular}

Legenda: QVT 1: remuneração e compensação; QVT 2: condições de trabalho; QVT 3: oportunidade imediata para uso e desenvolvimento das capacidades humanas; QVT 4: oportunidade futura de crescimento e segurança; QVT 5: integração social na organização do trabalho; QVT 6: constitucionalismo na organização do trabalho; QVT 7: trabalho e espaço total de vida; QVT 8: relevância social da vida no trabalho; QVT Global: avaliação global da qualidade de vida no trabalho. * $p \leq 0,001$. 
do QVT, embora 12 cruzamentos evidenciaram moderada correlação e 15 cruzamentos indicaram fraca correlação. A correlação muito fraca $\left(\mathrm{r}_{\mathrm{s}} 0,19\right)$ foi encontrada apenas entre as dimensões remuneração e compensação e trabalho e espaço total de vida.

Quanto às correlações entre as dimensões do EV e sua avaliação global (Tabela 4), observa-se forte correlação da avaliação global do EV com o componente atividade física $\left(\mathrm{r}_{\mathrm{s}} 0,60\right)$ e com o controle do estresse $\left(r_{s} 0,61\right)$. A moderada correlação foi demonstrada com as dimensões nutrição $\left(r_{s} 0,58\right)$ e relacionamentos $\left(r_{s}\right.$ 0,46). E a fraca correlação foi evidenciada com o componente comportamento preventivo $\left(\mathrm{r}_{\mathrm{s}} 0,38\right)$.

No que diz respeito às correlações entre os componentes do EV, constatou-se que o componente comportamento preventivo foi aquele que apresentou a correlação mais fraca com as demais dimensões. Por outro lado, os componentes atividade física e controle do estresse demonstraram as correlações mais fortes com os demais componentes. Além disso, cinco cruzamentos demonstraram fraca correlação e cinco cruzamentos indicaram muito fraca correlação entre as demais dimensões do EV.

Nas avaliações globais dos constructos QVT e EV, encontraram-se níveis de correlação muito fracos $\left(\mathrm{r}_{\mathrm{s}} 0,14\right)$ (Tabela 5). Apesar dos índices de correlação entre os componentes do EV e as dimensões da QVT evidenciarem correlação muito fraca em 38 cruzamentos, destaca-se que a dimensão trabalho e espaço total de vida e o componente controle do estresse obtiveram o maior índice de correlação $\left(r_{s}\right.$ 0,31). Além disso, a dimensão trabalho e espaço total de vida evidenciou fraca correlação com a avaliação global do EV $\left(\mathrm{r}_{\mathrm{s}} \mathrm{0,20}\right)$. O componente controle do estresse apresentou fraca correlação com a dimensão condições de trabalho $\left(\mathrm{r}_{\mathrm{s}} \mathrm{0}, 21\right)$ e a avaliação global da QVT ( $\mathrm{r}_{\mathrm{s}}$ 0,23).

\section{DISCUSSÃO}

Os resultados encontrados revelaram que os professores de Educação Física estão satisfeitos nos aspectos ligados às condições oferecidas para que o docente desenvolva suas capacidades, habilidades e conhecimentos; à progressão na carreira; aos seus direitos e deveres na escola; e à relevância social de sua função. Consequentemente, estes resultados influenciaram o desfecho da avaliação global da qualidade de vida no trabalho, ou seja, a cada dez docentes, seis estavam satisfeitos. Evidências semelhantes foram encontradas com docentes de

Tabela 4. Correlação entre os componentes e a avaliação global do estilo de vida.

\begin{tabular}{|c|c|c|c|c|c|c|}
\hline Variáveis & PEVI 1 & PEVI 2 & PEVI 3 & PEVI 4 & PEVI 5 & PEVI Global \\
\hline \multicolumn{7}{|l|}{ PEVI 1} \\
\hline PEVI 2 & $0,28^{*}$ & & & & & \\
\hline PEVI 3 & $0,18^{*}$ & $0,10^{* *}$ & & & & \\
\hline PEVI 4 & $0,17^{*}$ & $0,23^{*}$ & $0,09 * *$ & & & \\
\hline PEVI 5 & $0,27^{*}$ & $0,29 *$ & $0,15^{*}$ & $0,31^{*}$ & & \\
\hline PEVI Global & $0,58^{*}$ & $0,60^{*}$ & $0,38^{*}$ & $0,46^{*}$ & $0,61^{*}$ & \\
\hline
\end{tabular}

Legenda: PEVI 1: nutrição; PEVI 2: atividade física; PEVI 3: comportamento preventivo; PEVI 4: relacionamentos; PEVI 5: controle do estresse; PEVI Global: avaliação global do estilo de vida. ${ }^{*} p \leq 0,001,{ }^{* *} p \leq 0,01$.

Tabela 5: Correlações entre as dimensões e avaliação global da qualidade de vida no trabalho com os componentes e a avaliação global do estilo de vida.

\begin{tabular}{lccccccccc}
\hline Variáveis & QVT 1 & QVT 2 & QVT 3 & QVT 4 & QVT 5 & QVT 6 & QVT 7 & QVT 8 & QVT Global \\
\hline PEVI 1 & $0,12^{* *}$ & $0,09^{* * *}$ & 0,03 & 0,03 & $0,11^{* *}$ & 0,04 & $0,08^{* * *}$ & 0,04 & $0,08^{* * *}$ \\
PEVI 2 & 0,06 & $0,10^{* *}$ & $0,09^{* *}$ & 0,06 & $0,13^{*}$ & 0,06 & $0,18^{*}$ & $0,08^{* * *}$ & $0,13^{*}$ \\
PEVI 3 & 0,00 & 0,00 & 0,03 & 0,04 & 0,03 & 0,00 & $-0,01$ & 0,02 & 0,03 \\
PEVI 4 & $0,11^{* *}$ & $0,15^{*}$ & $0,13^{*}$ & $0,13^{*}$ & $0,17^{*}$ & $0,14^{*}$ & $0,17^{*}$ & $0,17^{*}$ & $0,18^{*}$ \\
PEVI 5 & $0,12^{* *}$ & $0,21^{*}$ & $0,19^{*}$ & $0,08^{* * *}$ & $0,16^{*}$ & $0,15^{*}$ & $0,31^{*}$ & $0,14^{*}$ & $0,23^{*}$ \\
PEVI Global & $0,10^{* * *}$ & $0,12^{*}$ & $0,12^{* *}$ & 0,07 & $0,15^{*}$ & $0,08^{* * *}$ & $0,20^{*}$ & $0,09^{* * *}$ & $0,14^{*}$
\end{tabular}

Legenda: QVT 1: remuneração e compensação; QVT 2: condições de trabalho; QVT 3: oportunidade imediata para uso e desenvolvimento das capacidades humanas; QVT 4: oportunidade futura de crescimento e segurança; QVT 5: integração social na organização do trabalho; QVT 6: constitucionalismo na organização do trabalho; QVT 7: trabalho e espaço total de vida; QVT 8: relevância social da vida no trabalho; QVT Global: avaliação global da qualidade de vida no trabalho; PEVI 1: nutrição; PEVI 2: atividade física; PEVI 3: comportamento preventivo; PEVI 4: relacionamentos; PEVI 5: controle do estresse; PEVI Global: avaliação global do estilo de vida. ${ }^{*} p \leq 0,001 * * p \leq 0,01 * * * p \leq 0,05$ 
Educação Física do sudoeste baiano ${ }^{18}$, e dos estados do Rio Grande do Sul ${ }^{2}$ e Santa Catarina ${ }^{3}$. Entretanto, os achados são contraditórios aos evidenciados pelos docentes da rede municipal de Porto Alegre/ $\mathrm{RS}^{19}$. Destaca-se que a percepção de satisfação nos componentes citados pode estar associada ao plano de carreira do magistério público estadual, o qual possibilita uma estabilidade e segurança profissional, além de valorizar o professor por meio de avanços progressivos no quadro funcional por tempo de serviço e cursos de aperfeiçoamento ${ }^{3}$.

Por outro lado, os professores demonstraram descontentamento nos assuntos relacionados à remuneração, condições de trabalho, integração social no ambiente de trabalho, e tempo equilibrado entre o lazer e o trabalho. De fato, investigações internacionais ${ }^{20-22}$ e nacionais ${ }^{2,3}$ têm demonstrado os mesmos motivos de insatisfação docente. A insatisfação com a infraestrutura deficitária, elevadas jornadas de trabalho, relacionamentos desgastados no ambiente de trabalho, e a falta de tempo para o lazer podem favorecer o surgimento doenças psíquicas e físicas, as quais interferem na vida pessoal do docente, além de influenciar negativamente na atividade laboral do professor $9,23-27$.

Sobre o EV, constatou-se que a maioria dos docentes investigados adota comportamentos positivos em relação ao comportamento preventivo, como o de acidentes, comportamento ético social, realização de exames preventivos periódicos e, os que dizem respeito à qualidade dos relacionamentos sociais, encontros com amigos para a prática de atividades de lazer, participação junto aos problemas da comunidade. Entretanto, os componentes: nutrição, atividade física e controle do estresse demonstraram ser os aspectos mais negativos do EV. Os comportamentos negativos parecem resultar da elevada jornada de trabalho, com a qual o professor busca melhorar suas condições financeiras em detrimento de hábitos saudáveis. Além disso, mesmo tendo três dos cinco componentes com índices negativos, evidenciou-se que seis de cada dez docentes de Educação Física apresentaram comportamentos positivos. Tais evidências são semelhantes às encontradas entre os docentes de Educação Física dos estados do Rio Grande do Sul ${ }^{2}$ e Santa Catarina ${ }^{11}$.

Sobre as correlações entre os indicadores das dimensões que compõem a QVT, constatou-se que remuneração e compensação e trabalho e espaço total de vida apresentaram os menores índices. Por outro lado, as dimensões que evidenciaram os maiores índices de correlação foram a relevância social da vida no trabalho e o constitucionalismo na organização do trabalho, as quais parecem compreender os indicadores mais simples e diretos para avaliação da QVT para este grupo.

Além disso, observam-se valores de correlação de fraca a moderada na análise pormenorizada da matriz analítica do QVT. Este achado parece refletir a restrição da temática abordada em suas dimensões, a qual avalia aspectos relacionados ao trabalho ${ }^{13}$

No constructo do EV, constatou-se que os componentes que têm o maior poder de predizer a avaliação global do EV são a atividade física e o controle do estresse. O componente comportamento preventivo apresentou o menor poder de predição do EV entre os professores de Educação Física. De fato, estudos têm evidenciado as associações entre nutrição, atividade física e estresse ${ }^{28}$. Entretanto, observa-se a necessidade de estudos que analisem as relações entre os comportamentos preventivos e relacionamentos com os demais componentes.

Apesar destes achados, torna-se importante destacar que os índices de correlação apresentados entre os componentes do constructo do EV foram considerados fracos ou muito fracos. Tais resultados parecem refletir a abrangência dos assuntos abordados na matriz de análise do EV ${ }^{15}$.

Ao avaliar os níveis de correlação entre os constructos da QVT e EV, identificou-se muito fraca e fraca correlação entre os temas abordados nas duas matrizes analíticas. No entanto, a evidência que os constructos demonstraram baixos níveis de correlação pode ser um indicativo substancial para confirmar a existência dos parâmetros individuais e socioambientais ${ }^{1}$, o que motiva a continuação das investigações nesta área.

Outro aspecto relevante é que alguns indícios nesta análise evidenciaram que há similaridade de opiniões entre o tempo dedicado entre lazer e trabalho com o controle do estresse; controle do estresse com as condições de trabalho; o controle do estresse e avaliação global do EV; e o tempo equilibrado entre lazer e trabalho com a avaliação global do QVT. De fato, os assuntos relacionados ao estresse e ao tempo dedicado ao lazer são aqueles que mais interferem ou são afetados pelo constructo diferente a que ele pertence. Alguns estudos evidenciam que a falta de equilíbrio do tempo dedicado ao lazer e ao trabalho contribui para o surgimento do quadro sintomático de estresse laboral ${ }^{9,26}$.

\section{CONCLUSÕES}

A partir dos resultados obtidos, conclui-se que a maioria dos professores de Educação Física da rede 
estadual pública de ensino do Paraná, está satisfeita com a QVT e com o EV, contudo, manifestaram insatisfação no que se refere aos fatores: remuneração e compensação, condições de trabalho, trabalho e espaço total de vida e índices preocupantes de comportamentos negativo nos componentes nutrição, atividade física e controle do estresse.

As correlações entre as dimensões da QVT e os componentes do PEVI foram consideradas muito fracas, o que evidencia determinada diferenciação entre os parâmetros socioambientais e individuais da qualidade de vida do trabalhador docente. No entanto, a atitude de precaução frente às evidências encontradas e também a carência de estudos na literatura consultada sobre o assunto, justificam a continuação dos estudos para aprofundar esta temática.

Além disso, as análises pormenorizadas das matrizes analíticas da QVT e do EV destacaram a maior correlação entre os componentes da QVT, uma vez que seu constructo aborda assuntos relacionados a uma temática específica (trabalho), enquanto que o EV obteve correlações fracas e muito fracas. Isto se deve ao fato dos elementos constituintes da matriz de análise do EV serem divergentes entre si.

As evidências encontradas realçam que, tanto nas avaliações globais da QVT quanto nas avaliações globais do EV, foram identificados os valores de maior correlação (moderada e forte) entre os componentes de seus respectivos constructos. Neste sentido, evidencia-se que mesmo não estando relacionadas diretamente entre si, as avaliações globais tanto do parâmetro socioambiental (QVT) quanto do parâmetro individual (EV), abordam o cotidiano do profissional docente dentro e fora do ambiente escolar e que tais aspectos podem auxiliar na avaliação e, consequentemente, na aplicação de programas que busquem a melhoria da QV deste profissional.

\section{REFERÊNCIAS BIBLIOGRÁFICAS}

1. Nahas MV. Atividade física, saúde e qualidade de vida: conceitos e sugestões para um estilo de vida ativo. 4 . ed. Londrina: Midiograf, 2006.

2. Lemos CAF, Nascimento JV, Borgatto AF. Parâmetros individuais e sócio-ambientais da qualidade de vida percebida na carreira docente em Educação Física, Rev bras Educ Fís Esp 2007;21(2):81-93.

3. Both J, Nascimento JV, Borgatto AF. Percepção da qualidade de vida no trabalho ao longo da carreira docente em educação física. Rev Bras Cineantropom Desempenho Hum 2008;10(4):372-8.

4. Folle A, Farias GO, Boscatto JD, Nascimento JV. Construção da Carreira Docente em Educação Física: Escolhas, Trajetórias e Perspectivas. Movimento 2009;15(1):25-49.
5. Nerrière E, Vercambre MN, Gilbert F, Masféty VK. Voice disorders and mental health in teachers: a cross-sectional nationwide study. BMC Public Health 2009;9:370-8.

6. Stockard J, Lehman MB. Influences on the Satisfaction and Retention of 1st-Year Teachers: The Importance of Effective School Management. Educ Adm Q 2004;40(5):742-71.

7. McCormack D, Casimir G, Djurkovic N, Yang L. The concurrent effects of workplace bullying, satisfaction with supervisor, and satisfaction with co-workers on affective commitment among schoolteachers in China. Int J Conflict Manag 2006;17(4):316-31.

8. Evers W, Tomic W, Brouwers A. Constructive thinking and burnout among secondary school teachers. Social Psychol Educ 2005;8(4):425-39.

9. Ozan MB. A study on primary schoolteacher burnout levels: the northern cyprus case. Education (Chula Vista) 2009;129(4):629-703.

10. Moreira HR, Farias GO, Both J, Nascimento JV. Qualidade de vida no trabalho e síndrome de burnout em professores de educação física do estado do Rio Grande do Sul, Brasil. Rev Bras Ativ Fís Saúde 2009;14(2):115-22.

11. Both J, Nascimento JV, Borgatto AF. Estilo de vida dos professores de Educação Física ao longo da carreira docente no estado de Santa Catarina, Rev Bras Ativ Fís Saúde 2007;12(3):54-64.

12. Both J, Nascimento JV, Lemos CAF, Donegá AL, Ramos MHKP, Petroski EC, Duarte MFS. Qualidade de vida no trabalho percebida por Professores de Educação Física. Rev Bras Cineantropom Desempenho Hum 2006;8(2):45-52.

13. Walton RE. Quality of working life: what is it? Sloan Manag Rev 1973;15 (1): 11-21.

14. Nahas MV, Barros MGV, Francalacci V. O pentáculo do bem estar: base conceitual para a avaliação do estilo de vida em indivíduos ou grupos. Rev Bras Ativ Fís Saúde 2000;2(5):48-59.

15. Both J, Borgatto AF, Nascimento JV, Sonoo CN, Lemos CAF, Nahas MV. Validação da escala "Perfil do Estilo de Vida Individual”. Rev Bras Ativ Fís Saúde 2008;13(1):5-14.

16. Lemos CAF. Qualidade de vida na carreira profissional de professores de Educação Física do magistério público estadual/RS. 2007. 144f. Dissertação (Mestrado em Educação Física) - Programa de Pós-Graduação em Educação Física, Centro de Desportos, Universidade Federal de Santa Catarina, Florianópolis, 2007.

17. Mitra A, Lankford S. Research methods in park, recreation and leisure services. Champaign: Sagamore Publishing; 1999.

18. Gomes AM, Borges JL, Nascimento JV. Ciclos de Desenvolvimento Profissional e a Qualidade de Vida de Professores de Educação Física da Região Sudoeste da Bahia. Rev Bras Educ Fís, Esporte, Lazer e Dança 2007;2 (4):104-14.

19. Wittizorecki ES, Molina Neto V. O trabalho docente dos professores de Educação Física na Rede Municipal de Ensino de Porto Alegre. Movimento 2005;11(1):47-70. 
20. Jabnoun N, Fook CY. Job satisfaction of secondary school teachers in Selangor, Malaysia. Int J Commerce e Manag 2001;11(3/4):72-90.

21. Nilan P. Teachers` work and schooling in Bali. Inte Rev Educ 2003; 49(6):563-84.

22. Papanastasiou EC, Zembylas M. Job satisfaction variance among public and private kindergarten school teachers in Cyprus. Int J Educ Res 2005;43(3): 147-67.

23. Araújo TM, Sena IP, Viana MAE, Araújo EM. Mal-Estar Docente: Avaliação de Condições de Trabalho e Saúde em Uma Instituição de Ensino Superior. Rev Baiana Saúde Pública 2005;29(1):6-21.

24. Crossman A, Harris P. Job Satisfaction of Secondary School Teachers. Educ Manag Adm Leadersh 2006;34(1): 29-46.

25. Carvalho AJFP, Alexandre NMC. Qualidade de vida e sintomas osteomusculares relacionados ao trabalho em professores do ensino fundamental. Rev Bras Fisioter 2006;10(1):35-41.

26. Valério FJ, Amorim C, Moser AM. A síndrome de Burnout em professores de Educação Física. Rev Psicol IMED 2009; 1 (1): 127-136.
27. Lipp MEN, Tanganelli MS. Stress e Qualidade de Vida em Magistrados da Justiça do Trabalho: Diferenças entre Homens e Mulheres. Psicol Reflex Crit 2002;15(3):537-48.

28. Barros MVG, Nahas MV. Comportamentos de risco, auto-avaliação do nível de saúde e percepção de estresse entre trabalhadores da indústria. Rev Saude Publica 2001;35(6):554-563.

\section{Endereço para correspondência}

Hudson de Resende Moreira

Rua Santo Antônio, 756 - Barreiros, CEP: 88.117-350 - São José, SC, Brasil E-mail: hudsonrm6@yahoo.com.br 